\title{
Assessment of Gastrointestinal Sensation - A Review
}

\author{
Anthony R. Hobson Qasim Aziz \\ Section of Gastrointestinal Sciences, Division of Medicine and Neurosciences - Hope, University of Manchester, \\ Hope Hospital, Salford, Lancs., UK
}

\section{Key Words}

Visceral hypersensitivity · Functional gastrointestinal disorders · Evoked potentials · Visceral pain · Outcome measures

\begin{abstract}
Understanding the mechanisms of symptoms in patients with gastrointestinal disorders remains a great challenge. One of the major problems facing clinicians in this area is the limited information gained from subjective outcome measures commonly used to assess these conditions. To address this, various stimulation and recording techniques, commonly used by neurologists, have been adapted to study gastrointestinal sensory processing. This review article provides an overview of this expanding research area and discusses the advantages and disadvantages of each approach.
\end{abstract}

Copyright (C) 2006 S. Karger AG, Basel

\section{Introduction}

Sensory signals arising from gastrointestinal (GI) afferents encode a wide range of peripheral activity and predominantly help regulate our homeostatic balance as well as modulating motility, secretion and appetite [1]. Most of these signals are subliminal, rarely reaching our consciousness; however, liminal sensations such as satiety and fullness, along with supraliminal sensations such as pain, complete this GI sensory spectrum.

A body of evidence which dates back centuries tells us that sensory information arising from the GI tract comprises one interoceptive component of a multifaceted neurobehavioral system involved in homeostasis, pain and emotional-motivational processes [2-4]. This is supported by recent data which demonstrate that GI sensations of all intensities invoke activity in a intricate array of cortical and subcortical structures via projections from both vagal and spinal afferents [3,5-8]. Whilst this is a fascinatingly complex area of research which transcends many biomedical disciplines, for the gastroenterologist it poses a surfeit of clinical problems.

The aim of this article is to provide a general overview of the techniques that have been used to assess GI sensation in both health and disease. However, instead of concentrating on some of the more traditional considerations in GI sensory testing, such as the biomechanical properties of the viscus in question, which have been reviewed in detail elsewhere [9], we shall consider GI sensory testing from both a neurological and mind-body perspective. In this way we can begin to address some of the limita-

\section{KARGER}

Fax +4161306 1234 E-Mail karger@karger.ch www.karger.com
Dr. Anthony R. Hobson

Section of Gastrointestinal Sciences, Division of Medicine and Neurosciences - Hope University of Manchester, Hope Hospital Salford, Lancashire M6 8HD (UK)

E-Mail Anthony.Hobson@manchester.ac.uk 
tions and pitfalls of different techniques and develop a rationale for improving the outcome measures that we use as primary end-points when assessing GI sensation.

\section{Definitions}

As we shall allude to the terms peripheral and central sensitization throughout this article, what follows is a brief definition of the mechanisms of these two hypersensitivity states. Peripheral sensitization of nerves within the gut can occur when inflammatory mediators, such as $\mathrm{K}^{+}, \mathrm{H}^{+}$, adenosine triphosphate, bradykinin and prostaglandins, activate and sensitize nociceptive afferent nerves following a transient inflammatory/injurious event [10].

Fibroblasts and mast cells within the inflammatory milieu contribute to nerve sensitization by releasing nerve growth factor which increases transcription of the neuropeptides substance $\mathrm{P}$ and calcitonin gene-related peptide at the neuronal cell body [10]. These are transported antegradely and are released by afferent terminals inducing further release of inflammatory mediators such as serotonin and histamine. This initiates additional nerve activation and the release of yet more substance P. Previously silent nociceptors become recruited and contribute to the development of hyperalgesia [10].

A secondary consequence of peripheral sensitization is the development of an area of hypersensitivity in the surrounding uninjured tissue. This phenomenon is due to changes in the activities of spinal afferents and is called central sensitization. Central sensitization is sustained by phosphorylation of N-methyl- $D$-aspartate receptors expressed in dorsal horn neurons [11, 12], which induces changes in the receptor kinetic properties and increases its sensitivity to synaptically released glutamate $[13,14]$. This leads to an increase in the excitability and the receptive fields of the spinal neurons, and results in recruitment and amplification of both non-nociceptive and nociceptive inputs from the adjacent healthy tissue [15].

\section{Stimulation Modalities}

The most important factor when designing an experimental protocol to assess GI sensation is to have a clear hypothesis regarding the biological question that is to be addressed. The GI tract contains afferents that encode a wide range of intensities and modalities of stimulation including electrical, mechanical, thermal and chemical (including nutrient) stimuli [1]. Choosing the correct stimulation method or interpreting differential responses to multiply stimuli may be extremely important in delineating pathological mechanisms. For instance, electrical stimulation is often thought of as non-physiological and therefore of limited use in assessing GI sensation. However, it is exactly because electrical stimulation bypasses the peripheral receptor that it is so useful, as it acts on the primary afferent directly allowing exploration of predominantly central afferent excitability [16]. In contrast, it is known that 'silent' nociceptors become active in inflammatory conditions [17] and the fact that electrical stimulation would activate these regardless of the presence or absence of a peripheral insult [16] renders this modality inadequate. If one wished to determine the presence of peripheral sensitization at the sensory receptor level in inflammatory conditions, then stimuli such as thermal, chemical and mechanical are the modalities of choice as has demonstrated in previous studies.

An excellent example of this type of approach comes from Chan et al. [18] in a study which aimed to examine the mechanism of rectal hypersensitivity in a cohort of patients with fecal urgency and incontinence. In this study the authors examined changes in the expression of the heat and capsaicin vanilloid receptor 1 (TRPV1 or VR1) in rectal sensory fibers, and correlated nerve fiber density with sensory abnormalities. As the TRPV1 receptor is activated by heat, the group designed a novel intrarectal thermode device to examine thermosensitivity in addition to a more traditional mechanical distension paradigm, and determined whether rectal hypersensitivity was modality-specific. The study showed that increases in TRPV1 correlated significantly with a decrease in rectal heat and distension thresholds leading the authors to conclude that fecal urgency and rectal hypersensitivity could result from increased numbers of polymodal sensory nerve fibers expressing TRPV 1 [18]. Whilst the study did not show modality-specific hypersensitivity, this is exactly the type of approach that has been used by neurologists in the study of neuropathic pain for many years and one which is increasingly being adopted in gastroenterology.

Drewes et al. [19] have taken this concept further by developing an intraesophageal catheter that is capable of providing mechanical, electrical, thermal (warm and cold) and chemical stimuli (fig. 1). This multimodal catheter has many advantages as it not only allows for the study of different receptor types but also nerve fiber types as cold signalling is predominantly an A $\delta$-fiber function whilst slow-ramp heat stimulation would be more suit- 
able to study unmyelinated C-fibers [16]. Interestingly, using this approach the authors were able to demonstrate modality-specific differences in sensory responses following experimental acidification of the esophagus, with subjects developing allodynia to thermal stimulation (hot and cold) and hyperalgesia to mechanical stimulation [19]. The slight disadvantage this catheter has is that the thermal stimulation requires distension of the balloon with fluid and therefore there may be a mechanical component during thermal sensory testing.

The past premise that the modality of stimulation used should aim to try and reproduce physiological events which may precipitate symptomatic episodes is a limiting one. For instance, we know that there is a poor correlation between gastroesophageal reflux events and symptoms and this is reflected in the poor sensitivity/specificity of sensory tests such as the Bernstein test [20, 21]. Therefore, adopting quantitative sensory testing approaches commonly used by neurologists in order to build a comprehensive picture of peripheral and central sensitivity may be a more beneficial approach allowing specific aberrant mechanisms of GI sensory processing to be identified and treatments targeted.

\section{Experimental Paradigms}

Small changes in the duration, frequency, site and intensity of stimulation can have dramatic effects on the sensory responses elicited from the GI tract and the information regarding the GI sensory system that is subsequently obtained. For instance, if stimulation frequencies $>0.3 \mathrm{~Hz}$ are used, temporal summation of the stimulus occurs leading to perceptual wind-up [22]. The mechanism of perceptual wind-up is similar to that responsible for central sensitization and occurs because spinal neuronal excitability or action potential output increases cumulatively with each successive stimulus due to an increase in the gain or amplification of the system [23].

We explored this phenomenon in a recent healthy human study in which we demonstrated the temporal summation occurs in the esophagus when a train of 20 electrical stimuli were given at a frequency of $2 \mathrm{~Hz}$ [24]. Interestingly, following a 30-min esophageal acid infusion and the subsequent induction of central sensitization and esophageal pain hypersensitivity, perceptual wind-up was enhanced, providing further evidence that esophageal acidification can lead to changes in the sensitivity of the central GI afferent pathway [24].

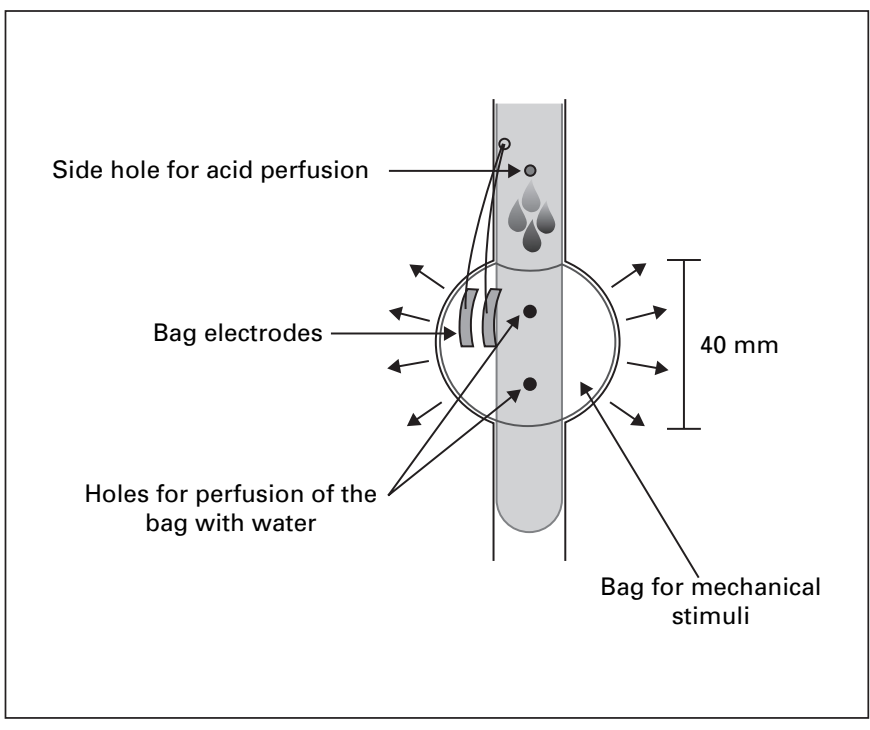

Fig. 1. Schematic illustration of the multimodal probe which provides electrical, mechanical, cold and warmth stimuli. The probe has a bag for mechanical and thermal stimuli, bag-mounted electrodes for electrical stimulation and a small infusion outlet proximal to the bag to allow chemical infusion. Reprinted from Drewes et al. [19], with permission.

The control frequency used in the above-mentioned study was $0.1 \mathrm{~Hz}$, a frequency which, if given in long trains, can be used to study the phenomenon of habituation. Habituation is thought to occur as a result of descending inhibitory influences from the brain, the result of which is a gradual increase in pain threshold over time. It has recently been speculated that the descending inhibitory system may be abnormal in patients with irritable bowel syndrome (IBS) and therefore, simply by altering the frequency of the experimental stimulus in the paradigm, it may be possible to examine two distinctly different aspects of GI sensory processing [25].

Sabate et al. [26] used a similar principle to examine two distinct receptor types in the rectum. In this study, rectal distension was the stimulus and this was applied using either a slow-ramp (40 $\mathrm{ml} / \mathrm{min})$ or rapid phasic $(900 \mathrm{ml} / \mathrm{min})$ protocol at four predetermined pressure values. It was speculated that slow-ramp distension preferentially activated sacral mucosal receptors whilst rapid phasic distension activated splanchnic afferents in the serosa and mesentery. These data were somewhat supported from subsequent studies using intrarectal lidocaine [26]. It is clear that these two types of stimulation paradigm evoke different psychophysical responses, and others have shown differential responses in IBS patients 
who demonstrate lower pain thresholds compared to controls for rapid but not slow-ramp distensions [27].

In order to further elucidate mechanisms of aberrant sensory processing, several groups have attempted to develop human models of GI sensitization. Sensitization commonly develops in response to a transient inflammatory or injurious event. In studies of somatic pain subcutaneous injection of chemical irritants or high-frequency trains of painful electrical stimuli have been used as sensitizing stimuli in human studies which have explored the acute mechanism of both peripheral and central sensitization [22].

Several groups have attempted to examine the effects of sensitizing stimuli on the subsequent processing of GI sensory information. One of the first studies to do this examined the effects of repeated high-pressure sigmoid distension on rectal sensory processing [28]. The hypothesis under test in this experiment stated that repetitive high-threshold stimulation of the sigmoid colon would induce activity-dependent neuronal plasticity within sacral spinal dorsal horn neurons. As spinal afferents from the sigmoid and rectum converge within the spinal cord, sensitization of spinal dorsal horn neurons within this convergent segment would result in a decrease in rectal pain thresholds. The results of this study showed that whilst in healthy subjects sigmoid distension had no effect on rectal pain thresholds, all IBS patients developed rectal hyperalgesia [28]. The physiological basis for using sigmoid distension was that IBS patients often demonstrated large amplitude sigmoid/colonic contractions and therefore repeated distension may somehow mimic the process by which increased afferent input into the spinal cord may lead to GI sensitization.

Whilst this study aimed to develop a clinically relevant model of IBS, Sarkar et al. [29] used a similar approach to understand the mechanisms of esophageal hypersensitivity in patients with non-cardiac chest pain (NCCP). In this study, the sensitizing stimulus used was hydrochloric acid $(0.15 M)$, which was chosen on the basis that in a proportion of patients symptoms are well controlled with acid suppression and therefore acid is implicated in the clinical condition to be modeled [30]. Pain thresholds were recorded in the proximal esophagus, a region not exposed to the acid infusion, and also on the area of somatic referral on the anterior chest wall [29].

Once again, these regions were chosen because, whilst there was no acid exposure and therefore no peripheral damage to these areas, both have convergent projections to the same spinal segments as the distal esophagus where the infusion took place [29]. Therefore, changes in pain thresholds from the upper esophagus and chest wall would provide information regarding the sensitivity of the central afferent pathway.

The results of this study showed that following a 30min acid infusion into the distal esophagus, pain thresholds in the upper esophagus and area of somatic referral reduced significantly in healthy subjects, and, importantly, this response was heightened in NCCP patients [29]. This provided important evidence to support the hypothesis that a proportion of patients with NCCP have changes within the spinal afferent system similar to that seen in patients with other certain types of somatic pain disorder. We have gone on to examine the important role that endogenous acid reflux plays in the development of chest pain and other groups have reproduced these data in healthy subjects $[19,31]$.

Hammer et al. [32, 33] have produced two studies which have looked at both the sensory aspects and latterly the sensitizing effects of capsaicin in the jejunum. Capsaicin is commonly used as a sensitizing stimulus in studies of somatic pain and in their initial study the authors expected to find that capsaicin induced a similar burning sensation when applied to the gut. However, they noted that in addition to a sensation of warmth and burning during the capsaicin infusion, sensory reports of cramping were also noted. The authors went on to show that this cramping sensation was independent of changes in small bowel motility or biomechanics and concluded that the capsaicin was acting directly on mechanosensitive afferents [32, 33]. In addition to capsaicin producing these unique sensory characteristics, it was also noted that the time to first sensation of a second capsaicin infusion was significantly reduced suggesting the induction of peripheral sensitization of capsaicin-sensitive afferents [32, 33].

In summary, when assessing GI sensation, one must not only consider the neurophysiological target of the stimulus, but also the mechanism which you wish to study. Manipulation of the environment in which a sensory stimulus is given, as has been done in many previous barostat-type studies, pre- and post-nutrient infusion for instance, can yield important physiological and pathophysiological information above and beyond that gained purely from quantitative sensory testing alone.

\section{What Outcome Measures Should Be Used?}

Perhaps the most important aspect of GI sensory assessment is the objectivity and robustness of the outcome measures used as primary end-points for the experiment. 
It is widely acknowledged that, if considered in isolation, many of these measures are limited. The real challenge for gastroenterologists is to consider how the mind and body interact to generate a sensation or symptom rather than to consider a single measure as the most salient factor in this process. To this end there has been an explosion of research in this area with many groups attempting to find the holy grail of identifying a robust, clinically significant, objective biological marker of GI hypersensitivity. Whether such a marker exists is debatable, but the following section outlines some of the more promising and, for completeness, unpromising approaches.

\section{Sensory Reports}

In an ideal world, it would be possible to distend the esophagus of a NCCP patient, ascertain the threshold at which this patient described pain and decide whether this patient had a 'sensitized' esophagus. The reality is that many other factors aside from the sensitivity of the afferent nerve contribute to our perception of a stimulus. Many studies in healthy subjects have shown that psychological interventions that alter baseline states of anxiety, mood, attention and emotion influence our perception of a given stimulus [34-36]. Consequently, when sensory reports are used as primary end-points, the data are vulnerable to the inherent response bias of such an approach [37].

To date, translational studies in, what in many cases will be a heterogeneous patient population with multiple etiologies for the same symptom, often tell us little about the underlying pathophysiology of the condition. This has not prevented researchers continuing in this vein; a fact that probably accounts for the disappointingly slow progress in understanding mechanisms for the development of symptoms and in developing effective treatments for conditions such as functional GI disorders [9, 38].

Examining the mechanisms of symptoms arising from the GI tract requires an almost forensic approach. Looking at the gut in isolation is a little like a crash investigator drawing conclusions from merely examining the wreckage, without taking into account the control mechanisms of the vehicle and the environmental factors leading up to, and following, the event. Additional information regarding the psychological state, central nervous system sensitivity, autonomic nervous system reactivity and hypothalamicpituitary-adrenal axis measures are now adding to our understanding of this complex and integrated system and a summary of some of these findings follows.

Assessment of Gastrointestinal Sensation

\section{Afferent Pathway Sensitivity}

Objectively assessing the sensitivity of extrinsic GI afferent pathways is desirable as it would allow clinicians to identify those patients with hypersensitive visceral afferents. To this end, commonly used neurophysiological techniques have been adapted allowing us to non-invasively investigate the integrity and characteristics of GI afferent pathways and slowly our knowledge in this area is expanding.

Neurophysiologists have used evoked potentials (EP) to study somatosensory, visual, auditory and pain pathways for half a century [39]. This technique involves the brief presentation of a sensory stimulus which is timeand phase-locked to the recording of the electroencephalogram (EEG) via surface electrodes placed on the scalp. The event-related signal is small in amplitude but occurs at the same moment in time following each stimulus, whilst the large amplitude background EEG occurs randomly. In order to extract the desired signal, repeated stimuli are given and the subsequent brain activity averaged. This reduces the unwanted EEG whilst enhancing the event-related EP [39].

Frieling et al. [40] were the first to record EP responses to esophageal stimulation and they, along with other investigators, suggested that the esophageal evoked response (EEP) was predominantly mediated via vagal afferent pathways. However, further studies have shown that the stimulus response characteristic of the visceral EP points to mediation via by both spinal and vagal afferents [41, 42]. Frieling et al. [43] also recorded these responses from the rectosigmoid, and subsequently it has been demonstrated that EPs can be recorded in response to stimulation throughout the GI tract [44].

A comparison of EEP elicited by electrical and mechanical stimulation showed that both responses were mediated by thinly myelinated A $\delta$-fibers, both produced responses of identical morphology (fig. 2) and that the latency difference between the first mechanical and electrical EEP component of $\sim 50 \mathrm{~ms}$ was due to the physical delay encountered during balloon inflation and not activation of different fiber types [42]. This is not to say that unmyelinated $\mathrm{C}$-fibers are not activated by esophageal stimulation, just that they do not contribute to the early response complex recorded with EEP [42].

Importantly, the amplitude of mechanically evoked EEP was smaller than that seen to electrical stimulation. It is known that the amplitude of the EEP increases with increased afferent recruitment. These amplitude differences could therefore be explained by the fact that me-

Dig Dis 2006;24:267-277 
Fig. 2. Raw data for mechanical and electrical stimulation in 6 subjects. Each trace represents the average of 200 esophageal stimuli acquired at a stimulation intensity that was $75 \%$ of the subjects' pain threshold. In order to demonstrate the similarities of morphology and inter-peak latencies, the authors have superimposed the traces and the P1 peaks have been aligned to compensate for the differences in peak latency. This also demonstrates the smaller amplitude CEP components acquired with mechanical stimulation. Reproduced from Hobson et al. [42], with permission.

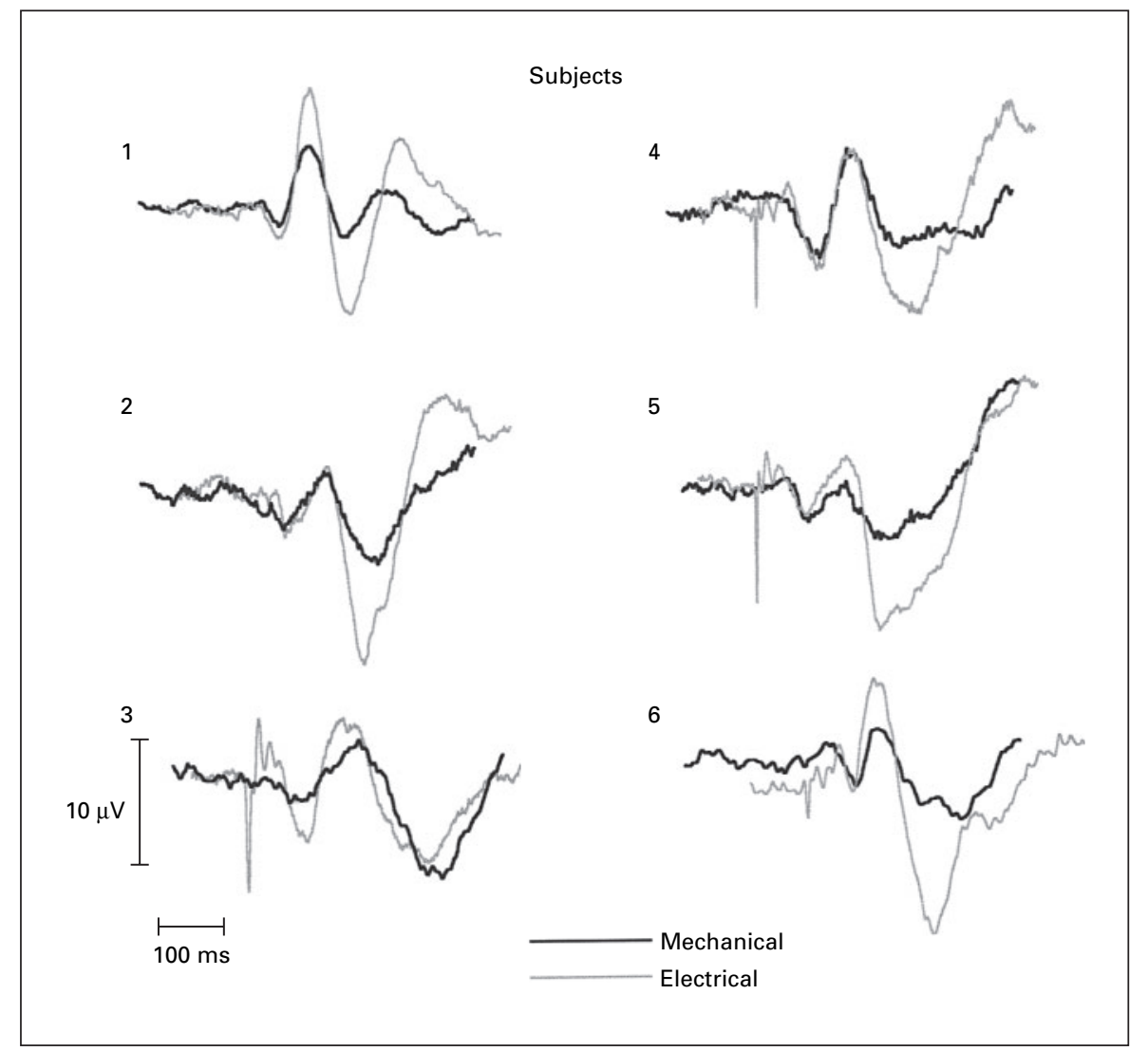

chanical stimulation is specific to mechanosensitive afferent receptors whilst electrical stimulation activated all afferents regardless of modality, hence leading to greater afferent recruitment [42].

Studies have shown that as stimulation intensity and sensory perception increased towards pain, there was an associated reduction in the latency and increase in amplitude of the EEP components [41, 42]. This phenomenon is common across all EP modalities and reflects the recruitment of an increasing number of afferents. This relationship between amplitude, latency and the intensity of sensory perception is an extremely important property, as visceral EP measures become objective neurophysiological correlates of GI sensory processing [45]. The ability to record objective neurophysiological measures which correspond directly to subjective pain ratings overcomes many of the limitations of previous studies of visceral hypersensitivity [46].

We have utilized these properties of the EP response to study mechanisms of esophageal hypersensitivity. First, using the esophageal acid sensitization model described briefly earlier, we recorded EEP prior to and fol- lowing the development of esophageal sensitization using electrical stimulation [47].

This study showed that whilst using the same intensity stimulus at both time points, following acid, subjects perceived the stimulus more intensely and this was associated with a reduction in the EEP latency (fig. 3). This provided strong evidence to support the hypothesis that the reduction in pain threshold seen in response to experimental esophageal acidification was associated with changes in the sensitivity of the central afferent pathway. We have now begun to translate these findings into a population of patients with NCCP and have indeed shown that a subset of these patients (approx. 35\%) have neurophysiological profiles consistent with increases in central afferent pathway sensitivity [48]. However, just as importantly, we have identified a second subset of NCCP patients that have identical clinical and psychophysical characteristics in association with esophageal hypersensitivity, but have distinctly different neurophysiological profiles. The delayed latency EEP components seen in this second group of patients points to the primary mechanism of hypersensitivity not lying in the afferent path- 
Fig. 3. Effect of lower esophageal acid or saline infusion on latency of EEP acquired from the upper esophagus. The top trace $(\mathbf{A})$ demonstrates a reduction in latency of $\mathrm{N} 1$ and $\mathrm{P} 2$ components evoked by stimulation of the upper esophagus following acid infusion in the lower esophagus. The bottom trace $(\mathbf{B})$ acquired from the same region following saline infusion show no changes in the EEP response. Reproduced from Sarkar et al. [47], with permission.

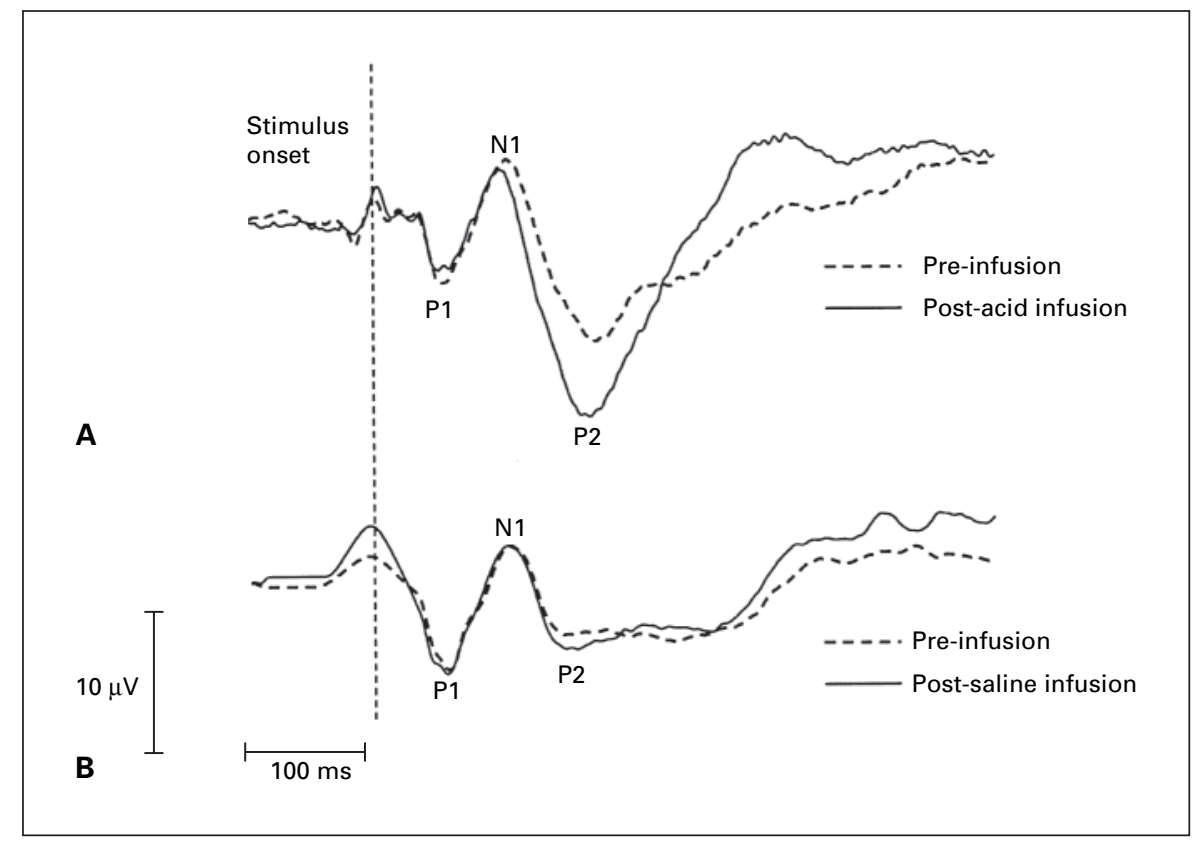

way, as afferent transmission is normal, but in the secondary processing of GI sensory signals in the brain. It is possible that this group of patients have a predominantly psychological cause for their symptoms, a factor which would indicate a different treatment and management strategy to those with sensitized esophageal afferents [48].

\section{Functional Brain Imaging}

Metabolic neuroimaging techniques such as positron emission tomography (PET) and functional magnetic resonance imaging (fMRI) have revealed that, following stimulation of the GI tract with subliminal, liminal and supraliminal stimuli, activity is observed within a broad network of cortical and subcortical structures [49, 50]. PET and fMRI rely on the principle that increased neuronal activity within the brain is associated with increased metabolism and regional cerebral blood flow [51, 52]. Comparison of images recorded during resting and active periods reveals regions of increased cortical activity and these measures have been used to increase understanding of the functional properties of the brain.

A recent meta-analysis of functional brain imaging studies of GI sensation revealed the GI tract is represented in all of the major cortical regions activated by somatic stimuli, i.e., primary somatosensory cortex (S1), sec- ondary somatosensory cortex (S2), insula, cingulate, prefrontal cortex and motor cortex [8]. Activation of subcortical regions such as the thalamus and periaqueductal gray have also been demonstrated with PET [50]; however, at the time of writing, only one fMRI study has shown convincing subcortical activation [53]. Despite being few in number, these studies show relatively good concordance giving us confidence that they are an accurate representation of GI sensory-cortical processing.

To give one example of how brain imaging can help our understanding of GI sensory processing, several studies have examined differences between esophageal and somatic pain processing $[53,54]$. The most recent of these studies performed by Strigo et al. [53] compared cortical activity generated by distension of the esophagus and thermal stimulation of the anterior chest wall. In this study, both stimuli were matched psychophysical for intensity, however, esophageal stimulation was perceived to be more unpleasant than cutaneous stimulation and the major differences in cortical representation occurred in S1, insula and pre-frontal cortex.

Of additional interest was the fact both esophageal and chest wall stimulation activated $\mathrm{S} 1$ in the region associated with the homuncular representation of the trunk; however, esophageal stimulation alone activated the more lateral aspects of S1 often referred to as gustatory region of $\mathrm{S} 1$. The authors concluded that this diffuse representation of the esophagus in $\mathrm{S} 1$ was consistent with the 


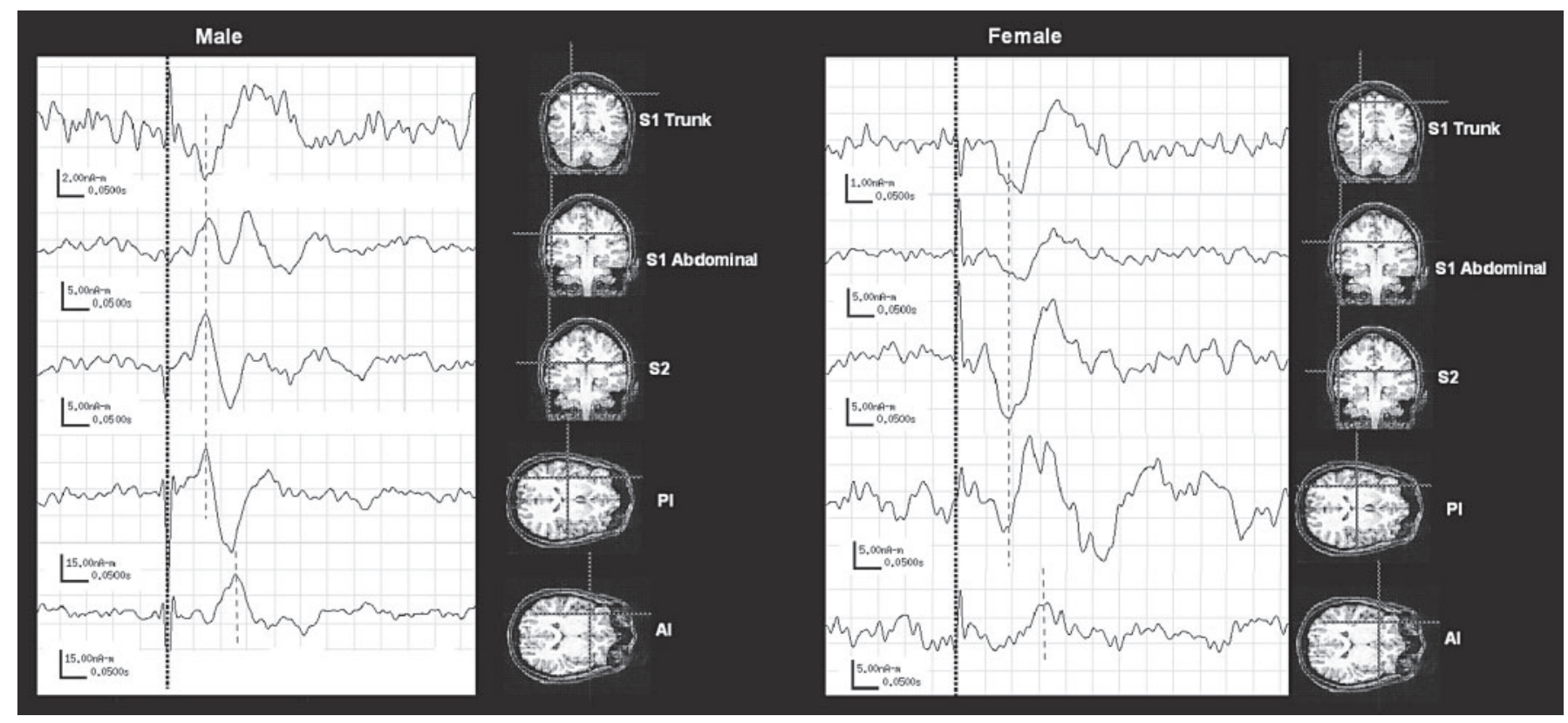

Fig. 4. MEG responses from 1 male (left) and 1 female (right) subject taken from representative positions placed in $\mathrm{S} 1, \mathrm{~S} 2$ and insula cortices. It can be seen that the first peak (P1, either positive or negative) in the first four cortical regions occurs at the same latency. However, the first peak in the anterior insula (bottom trace) has a longer latency. This was consistent for both male and female subjects. Reprinted from Hobson et al. [59], with permission.

fact that visceral pain can be referred to the skin but not vice versa [53].

The hope of many researchers in this field has been that brain imaging would provide the kind of objective neural correlates needed to distinguish between different aberrant mechanisms of GI sensory processing in conditions like functional GI disorders. Unfortunately, other studies have shown that the brain activation patterns are dramatically altered depending on many environmental factors. For instance, in a classical conditioning paradigm using fMRI, the authors showed that similar brain regions were activated both in response to a stimulus but worryingly also in anticipation of the stimulus [34]. The fact that the outcome measure is not specific to the stimulus provides similar problems to those encountered with sensory reporting [46].

We have used another functional brain imaging technique, magnetoencephalography (MEG), to record esophageal cortical neuronal activity [55-57]. MEG detects the minute magnetic fields generated by groups of active cortical neurons using highly sensitive sensors. Unlike the electrical signal recorded with EP, which is distorted by all of the structures that lie between the cortical source and recording electrode, the magnetic field generated by an active group of cortical neurons passes through these structures without attenuation [58]. The major advantage of using MEG therefore is that it we can study specific brain regions in a similar manner to PET and fMRI; however, we can record changes in brain activity dynamically in real-time.

Figure 4 shows the type of responses we can record with MEG and these data have revealed that the earliest cortical activity to be detected following electrical stimulation of the esophagus occurs in a region that processes sensory discriminatory aspects of esophageal sensation (S1/S2), whilst secondary processing occurs in the cingulate approximately $30 \mathrm{~ms}$ following activation of S1/S2 [59]. As the signal detected with MEG is the magnetic component of the electrical field recorded with EEP, it can be used to temporally correlate activity occurring within specific cortical regions with the scalp recorded EEP. This therefore allows us to say that the earliest EEP component (P1), which occurs approximately $80-110 \mathrm{~ms}$ following esophageal stimulation, is likely to reflect activation of cortical regions involved in processing pain sensation, whilst later components $(\mathrm{N} 1, \mathrm{P} 2)$ are an amalgamation of cortical activity within regions that process both pain sensation and affect. It is clear therefore that 
these later components represent secondary processing of esophageal sensation/pain. Because the early MEG responses are stimulus-specific, this type of approach has a greater potential for becoming a robust and objective outcome measure to study GI sensory processing.

\section{Nociceptive Withdrawal (RIII) Reflex}

The nociceptive withdrawal reflex or RIII reflex is a physiological, polysynaptic reflex allowing for painful stimuli to activate an appropriate withdrawal response. The RIII reflex is easily measurable in an experimental and clinical setting, and is a reliable and objective tool for measurement of an individual's pain experience [60]. It is thought that the magnitude of the RIII reflex may be an important measure of spinally mediated nociceptive processing, and provide some insights into the sensitivity of this system [61].

The RIII reflex can be recorded from all four limbs; however, lower limb stimulation is the most commonly used technique. Standard testing includes transcutaneous electrical stimulation of the sural nerve in the retromalleolar space and recording of the impulse from the surface of the short head of the biceps femoris muscle, ipsilateral to the stimulated side [61]. Several groups have examined changes in the characteristics of the RIII reflex in response to either conventional stimulation of the GI tract or in response to a sensitizing stimulus [19, 26, 62, 63]. These studies have shown that depending on the type of stimulus used and the clinical condition under investigation, the RIII reflex can either be facilitated or inhibited [19, 26, 62, 63].

Bouhassira et al. [63] demonstrated that rapid rectal distensions facilitated the RIII reflex recorded from the lower limb, but at the highest distension level, facilitation was followed by inhibition. Slow-ramp rectal distension induced gradual inhibition of the RIII reflex, which correlated with both distension volume and visceral sensation. These data were confirmed in later studies and recently, Coffin et al. [62] have applied this methodology to patients with IBS. In this study the authors showed that in IBS patients, the RIII reflex was significantly facilitated during slow-ramp distension and that inhibitions induced by rapid distensions were significantly reduced and this was demonstrated without any differences between the volumes of distension and rectal compliance between groups (fig. 5). The group concluded that these results provided direct evidence that a hyperexcitability of spinal nociceptive processes is present in a large sub-

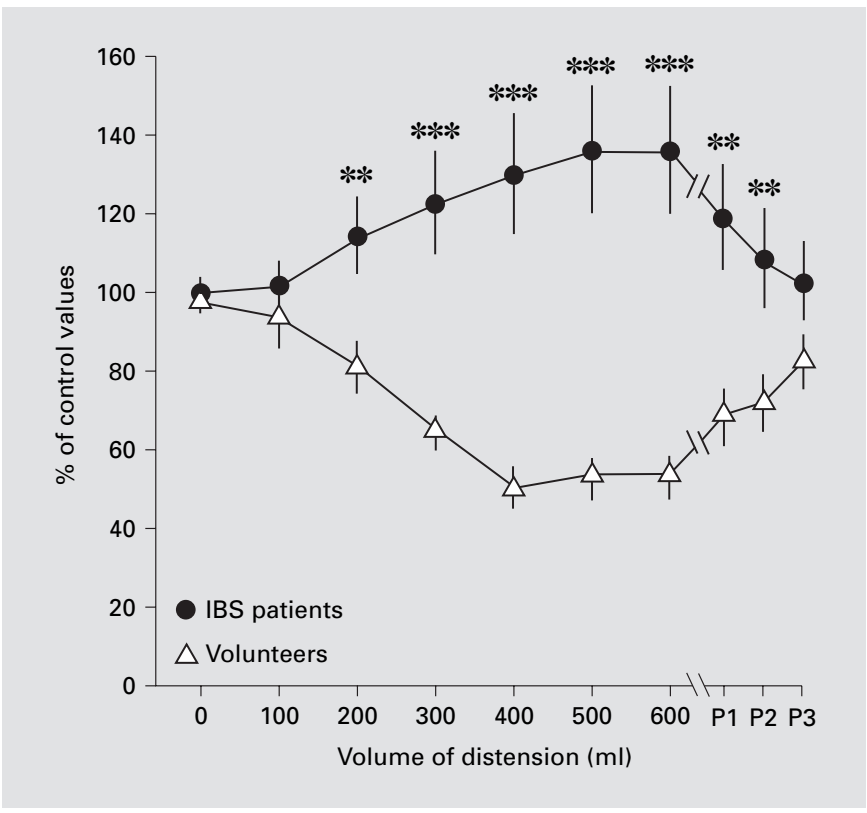

Fig. 5. Cumulative results showing the effects of slow-ramp distension on the RIII reflex in healthy volunteers and IBS patients. In healthy volunteers, slow-ramp distension induced a progressive decrease in the RIII with a progressive return to baseline after the end of distension. In IBS patients, the effects of slow-ramp distension were significantly different to those observed in volunteers as it induced progressive facilitation of the RIII reflex. $* * p=0.01$; $* * * p=0.001$. Reproduced from Coffin et al. [62], with permission.

group of IBS patients [62]. However, once again, interpretation of these data should be guarded. Studies have shown that factors such as sustained attention, anticipation of pain, sustained muscle contraction, the type of which may occur when contracting the anal sphincter during rectal distension, hypnosis and many others can have either facilitatory or inhibitory effects on RIII and therefore these must be taken into account if the outcome measure is to be robust.

\section{Autonomic Nervous System}

In man, autonomic nervous system (ANS) measures such as cardiac sensitivity to the baroreflex (CSB - afferent limb of the ANS) and cardiac vagal tone (CVT - efferent limb of the ANS), have, until recently, required off-line analysis of data acquired over a 60- to 90 -second period $[64,65]$. The limitation of this approach was highlighted in a recent study which investigated CSB responses to rectal distension in IBS patients. 
The study revealed that IBS patients exhibited a raised CSB relative to healthy controls whereas the converse was anticipated [65]. One explanation for this is that the method of CSB measurement used was limited to a 90-second time window whereas we know that the ANS is a dynamic system which fluctuates in its activity from second to second. In some recent pilot work, we have demonstrated that over a 60 -second period time frame both reductions and exaggerations of CSB and CVT are seen in response to esophageal distension (unpubl. data). These fluctuations are masked when using a poorer temporal resolution which may result in a false impression of a solitary rise in the ANS measure of interest. This would subsequently lead to an incorrect interpretation of results. Therefore, whilst the information gleaned from studies of ANS activity and reactivity to date has been limited, new advances in technology may give us the opportunity to expand on these experiments in a degree of detail not previously possible.

\section{Summary and Future Considerations}

This article has provided a general overview of some of the most recent advances in GI sensory testing. This is a very complex area and developing and understanding of the multiple systems involved in the generation of sensations and symptoms that may (or may not) arise from activity within the GI tract is a great challenge. As always, the technology is improving and we are able to stimulate the gut in many different ways and record online measures of central nervous system and ANS activity which will provide more robust outcome measures for these experiments. Hopefully, this will enable us to better understand the mechanisms of GI sensory dysfunction and develop more effective treatment strategies.

\section{References}

1 Sengupta JN, Gebhart GF: Gastrointestinal afferent fibres and sensation; in Holle GE (ed): Physiology of the Gastrointestinal Tract. New York, Raven Press, 1992, pp 483-519.

2 Cannon WB: The influence of emotional states on the functions of alimentary tract. Am J Med 1909; 137:480-487.

-3 Saper CB: The central autonomic nervous system: conscious visceral perception and autonomic pattern generation. Annu Rev Neurosci 2002;25:433-469.

4 Craig AD: Interoception: the sense of the physiological condition of the body. Curr Opin Neurobiol 2003; 13:500-505.

$\checkmark 5$ Willis WD, Westlund KN: Neuroanatomy of the pain system and of the pathways that modulate pain. J Clin Neurophysiol 1997;14:231.

-6 Willis WD, Al-Chaer ED, Quast MJ, Westlund $\mathrm{KN}$ : A visceral pain pathway in the dorsal column of the spinal cord. Proc Natl Acad Sci USA 1999;96:7675-7679.

7 Berntson GG, Sarter M, Cacioppo JT: Ascending visceral regulation of cortical affective information processing. Eur J Neurosci 2003; 18 : 2103-2109.

8 Derbyshire SW: A systematic review of neuroimaging data during visceral stimulation. Am J Gastroenterol 2003;98:12-20.

9 Camilleri M: Testing the sensitivity hypothesis in practice: tools and methods, assumptions and pitfalls. Gut 2002;51(suppl 1):i34-i40.

10 Woolf CJ, Salter MW: Neuronal plasticity: increasing the gain in pain. Science 2000;288: 1765-1769.
11 Treede RD, Magerl W: Multiple mechanisms of secondary hyperalgesia. Prog Brain Res 2000;129:331-341.

12 Woolf CJ: Evidence for a central component of post-injury pain hypersensitivity. Nature 1983; 306:686-688.

13 Chen A, Dworkin S, Haug J, Gehrig J: Human pain responsivity in a tonic pain model: psychological determinants. Pain 1989;37:143.

14 Chen L, Huang LY: Protein kinase C reduces $\mathrm{Mg}^{2+}$ block of NMDA-receptor channels as a mechanism of modulation. Nature 1992;356: 521-523.

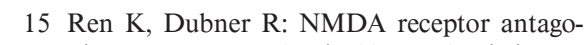
nists attenuate mechanical hyperalgesia in rats with unilateral inflammation of the hindpaw. Neurosci Lett 1993;163:22-26.

16 Graven-Nielsen T, Sergerdahl M, Svensson P, Arendt-Neilsen L: Methods for induction and assessment of pain in humans with clinical and pharmacological examples; in Kruger L (ed): Methods in Pain Research. Florida, CRC Press, 2001, pp 263-289.

17 Gebhart GF: Peripheral contributions to visceral hyperalgesia. Can J Gastroenterol 1999; 13(suppl A):37a-41a.

18 Chan C, Facer P, Davis J, Smith G, Egerton J, Bountra C, et al: Sensory fibres expressing capsaicin receptor TRPV1 in patients with rectal hypersensitivity and faecal urgency. Lancet 2003:361:385-391.

19 Drewes AM, Schipper KP, Dimcevski G, Petersen $\mathrm{P}$, Andersen OK, Gregersen $\mathrm{H}$, et al: Multi-modal induction and assessment of allodynia and hyperalgesia in the human oesophagus. Eur J Pain 2003;7:539-549.
20 Ghillebert G, Janssens J, Vantrappen G, Nevens F, Piessens J: Ambulatory 24-hour intraoesophageal $\mathrm{pH}$ and pressure recordings $\mathrm{v}$ provocation tests in the diagnosis of chest pain of oesophageal origin. Gut 1990;31:738-744.

21 Hewson EG, Sinclair JW, Dalton CB, Wu WC, Castell DO, Richter JE: Acid perfusion test: does it have a role in the assessment of noncardiac chest pain? Gut 1989;30:305-310.

22 Magerl W, Wilk SH, Treede RD: Secondary hyperalgesia and perceptual wind-up following intradermal injection of capsaicin in humans. Pain 1998; 74:257-268

23 Woolf CJ: Windup and central sensitization are not equivalent. Pain 1996;66:105-108.

24 Sarkar S, Woolf CJ, Hobson AR, Thompson DG, Aziz Q: Perceptual wind-up in the human oesophagus is enhanced by central sensitization. Gut 2006 (in press).

- 25 Hollerbach S, Kamath MV, Fitzpatrick D, Shine G, Fallen E, Upton AR, et al: The cerebral response to electrical stimuli in the oesophagus is altered by increasing stimulus frequencies. Neurogastroenterol Motil 1997;9: 129-139.

-26 Sabate JM, Coffin B, Jian R, Le Bars D, Bouhassira D: Rectal sensitivity assessed by a reflexologic technique: further evidence for two types of mechanoreceptors. Am J Physiol 2000;279:G692-G699.

-27 Mertz H, Naliboff B, Munakata J, Niazi N, Mayer EA: Altered rectal perception is a biological marker of patients with irritable bowel syndrome. Gastroenterology 1995;109:40-52. 
-28 Munakata J, Naliboff B, Harraf F, Kodner A, Lembo T, Chang L, et al: Repetitive sigmoid stimulation induces rectal hyperalgesia in patients with irritable bowel syndrome. Gastroenterology 1997;112:55-63.

-29 Sarkar S, Aziz Q, Woolf CJ, Hobson AR, Thompson DG: Contribution of central sensitisation to the development of non-cardiac chest pain. Lancet 2000;356:1154-1159.

- 30 Bautista J, Fullerton H, Briseno M, Cui H, Fass $\mathrm{R}$ : The effect of an empirical trial of high-dose lansoprazole on symptom response of patients with non-cardiac chest pain - a randomized, double-blind, placebo-controlled, crossover trial. Aliment Pharmacol Ther 2004; 19:11231130.

- 31 Sarkar S, Thompson DG, Woolf CJ, Hobson AR, Millane T, Aziz Q: Patients with chest pain and occult gastroesophageal reflux demonstrate visceral pain hypersensitivity which may be partially responsive to acid suppression. Am J Gastroenterol 2004;99:1998-2006.

- 32 Hammer J, Hammer HF, Eherer AJ, Petritsch W, Holzer P, Krejs GJ: Intraluminal capsaicin does not affect fluid and electrolyte absorption in the human jejunum but does cause pain. Gut 1998;43:252-255.

-33 Schmidt B, Hammer J, Holzer P, Hammer HF: Chemical nociception in the jejunum induced by capsaicin. Gut 2004;53:1109-1116.

-34 Yaguez L, Coen S, Gregory LJ, Amaro E, Altman $\mathrm{C}$, Brammer MJ, et al: Brain response to visceral aversive conditioning: a functional magnetic resonance imaging study. Gastroenterology 2005; 128:1819-1829.

- 35 Gregory LJ, Yaguez L, Williams SC, Altmann C, Coen SJ, Ng V, et al: Cognitive modulation of the cerebral processing of human oesophageal sensation using functional magnetic resonance imaging. Gut 2003;52:1671-1677.

- 36 Phillips ML, Gregory LJ, Cullen S, Cohen S, $\mathrm{Ng} \mathrm{V}$, Andrew C, et al: The effect of negative emotional context on neural and behavioural responses to oesophageal stimulation. Brain 2003;126:669-684.

- 37 Whitehead WE, Palsson OS: Is rectal pain sensitivity a biological marker for irritable bowel syndrome: psychological influences on pain perception. Gastroenterology 1998;115:12631271.

38 Camilleri M: Drugs targeting functional bowel disorders: lessons from drug trials. Curr Opin Pharmacol 2002;2:684-690.

39 Lewine JD, Orrison W: Clinical electroencephalography and event-related potentials; in Orrison W, Lewine J, Sanders J, Hartshone M (eds): Functional Brain Imaging. St Louis, Mosby, 1995, pp 327-368.
40 Frieling T, Enck P, Wienbeck M: Cerebral responses evoked by electrical stimulation of the esophagus in normal subjects. Gastroenterology 1989;97:475-478.

41 Hollerbach S, Kamath MV, Chen Y, Fitzpatrick D, Upton AR, Tougas G: The magnitude of the central response to esophageal electrical stimulation is intensity dependent. Gastroenterology 1997; 112:1137-1146.

42 Hobson AR, Sarkar S, Furlong PL, Thompson DG, Aziz Q: A cortical evoked potential study of afferents mediating human esophageal sensation. Am J Physiol 2000;279:G139-G147.

43 Frieling T, Enck P, Wienbeck M: Cerebral responses evoked by electrical stimulation of rectosigmoid in normal subjects. Dig Dis Sci 1989;34:202-205.

44 Hobday DI, Hobson AR, Sarkar S, Furlong PL, Thompson DG, Aziz Q: Cortical processing of human gut sensation: an evoked potential study. Am J Physiol 2002;283:G335-G339.

45 Hobson AR, Aziz Q: Brain processing of esophageal sensation in health and disease. Gastroenterol Clin North Am 2004;33:6991.

46 Hobson AR, Aziz Q: Brain imaging and functional gastrointestinal disorders: has it helped our understanding? Gut 2004;53:1198-1206.

47 Sarkar S, Hobson AR, Furlong PL, Woolf CJ, Thompson DG, Aziz Q: Central neural mechanisms mediating human visceral hypersensitivity. Am J Physiol 2001;281:G1196G1202.

48 Hobson AR, Furlong PL, Sarkar S, Mathews PJ, Willert RP, Worthen SF, Unsworth BJ, Azis Q: Neurophysiological assessment of esophageal sensory processing in noncardiac chest pain. Gastroenterology 2006;130(1):80 88.

49 Kern MK, Shaker R: Cerebral cortical registration of subliminal visceral stimulation. Gastroenterology 2002;122:290-298.

50 Aziz Q, Andersson JL, Valind S, Sundin A, Hamdy S, Jones AK, et al: Identification of human brain loci processing esophageal sensation using positron emission tomography. Gastroenterology 1997;113:50-59.

51 Sanders J, Orrison W: Functional magnetic resonance imaging; in Orrison $\mathrm{W}$, Lewine $\mathrm{J}$, Sanders J, Hartshone M (eds): Functional Brain Imaging. St Louis, Mosby, 1995, pp 239 326.

52 Hartshorne M: Positron emission tomography; in Orrison W, Lewine J, Sanders J, Hartshone M (eds): Functional Brain Imaging. St Louis, Mosby, 1995, pp 187-212.

53 Strigo IA, Duncan GH, Boivin M, Bushnell MC: Differentiation of visceral and cutaneous pain in the human brain. J Neurophysiol 2003 ; 89:3294-3303.
54 Aziz Q, Thompson DG, Ng VW, Hamdy S, Sarkar S, Brammer MJ, et al: Cortical processing of human somatic and visceral sensation. $\mathrm{J}$ Neurosci 2000;20:2657-2663.

55 Furlong PL, Aziz Q, Singh KD, Thompson DG, Hobson A, Harding GF: Cortical localisation of magnetic fields evoked by oesophageal distension. Electroencephalogr Clin Neurophysiol 1998;108:234-243.

56 Hecht M, Kober H, Claus D, Hilz M, Vieth J, Neundorfer B: The electrical and magnetical cerebral responses evoked by electrical stimulation of the esophagus and the location of their cerebral sources. Clin Neurophysiol 1999;110: 1435-1444.

-57 Schnitzler A, Volkmann J, Enck P, Frieling T, Witte OW, Freund HJ: Different cortical organization of visceral and somatic sensation in humans. Eur J Neurosci 1999; 11:305-315.

58 Lewine JD, Orrison W: Magnetoencephalography and magnetic source imaging; in Orrison W, Lewine J, Sanders J, Hartshone M (eds): Functional Brain Imaging. St Louis, Mosby, 1995, pp 369-417.

- 59 Hobson AR, Furlong PL, Worthen SF, Hillebrand A, Barnes GR, Singh KD, et al: Realtime imaging of human cortical activity evoked by painful esophageal stimulation. Gastroenterology 2005;128:610-619.

60 Skljarevski V, Ramadan NM: The nociceptive flexion reflex in humans. Pain 2002;96:3-8.

61 Garcia-Larrea L, Mauguiere F: Electrophysiological assessment of nociception in normals and patients: the use of nociceptive reflexes. Electroencephalogr Clin Neurophysiol Suppl 1990;41:102-118.

62 Coffin B, Bouhassira D, Sabate JM, Barbe L, Jian R: Alteration of the spinal modulation of nociceptive processing in patients with irritable bowel syndrome. Gut 2004;53:14651470.

63 Bouhassira D, Sabate JM, Coffin B, Le Bars D, Willer JC, Jian R: Effects of rectal distensions on nociceptive flexion reflexes in humans. Am J Physiol 1998;275:G410-G417.

64 Tougas G, Spaziani R, Hollerbach S, Djuric V, Pang C, Upton AR, et al: Cardiac autonomic function and oesophageal acid sensitivity in patients with non-cardiac chest pain. Gut 2001;49:706-712.

65 Van der Veek PP, Swenne CA, van de Vooren $\mathrm{H}$, Schoneveld AL, Maestri R, Masclee AA: Viscerosensory-cardiovascular reflexes: altered baroreflex sensitivity in irritable bowel syndrome. Am J Physiol Regul Integr Comp Physiol 2005;289:R970-R976. 